\title{
Synergistic neuroprotective effect of microglial-conditioned media treated with geniposide and ginsenoside Rg1 on hypoxia injured neurons
}

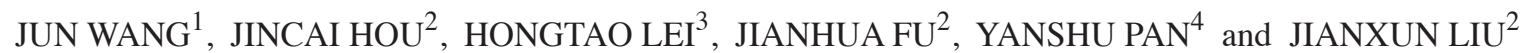 \\ ${ }^{1}$ Experiment Center, Institute of Basic Theory, Academy of Chinese Medical Sciences, Beijing 100700; \\ ${ }^{2}$ Institute of Basic Medical Sciences of Xiyuan Hospital, Beijing Key Laboratory of Pharmacology of Chinese Materia Medica, \\ Beijing 100091; ${ }^{3}$ Morphology Laboratory, The Experimental Research Center, Academy of Chinese Medical Sciences, \\ Beijing 100700; ${ }^{4}$ Graduate School, Beijing University of Chinese Medicine, Beijing 100029, P.R. China
}

Received October 9, 2014; Accepted June 26, 2015

DOI: $10.3892 / \mathrm{mmr} .2015 .4094$

\begin{abstract}
The synergistic mechanism underlying the effects of multi-component combined drug use for complex diseases remains to be fully elucidated. Microglial activation following ischemia can either affect neural survival or cause neuronal injury. The aim of the present study was to determine the synergistic effect of geniposide and ginsenoside $\mathrm{Rg} 1$, based on microglial-neuronal communication. N2a neuronal cells were divided into the following seven groups: Control group; normal cultured microglial cells in conditioned medium (N-MG-CM) group; oxygen-glucose deprivation (OGD) model group; OGD-injured MG-CM (I-MG-CM) group; geniposide-treated MG-CM (G-MG-CM) group; ginsenoside Rg1-treated MG-CM (R-MG-CM) group; and combination-treated MG-CM (C-MG-CM) group. A series of assays were used to detect the effects of the different MG-CM on neurons in terms of: (i) cell viability, determined using a Cell Counting Kit-8; (ii) lactate dehydrogenase (LDH) leakage rate; (iii) expression of NMDAR1 and activated caspase-3, detected using western blotting; (iv) mitochondrial transmembrane potential, determined by JC-1; and (v) mitochondrial ultrastructural features, determined using electron microscopy. The experimental results demonstrated that MG-CM including the integrated use of geniposide and ginsenoside $\mathrm{Rg} 1$ significantly
\end{abstract}

Correspondence to: Dr Jianxun Liu, Institute of Basic Medical Sciences of Xiyuan Hospital, Beijing Key Laboratory of Pharmacology of Chinese Materia Medicine, 1 Xiyuan Caochang, Haidian, Beijing 100091, P.R. China

E-mail: ljxunljxun@163.com; liujx0324@sina.com

Dr Jun Wang, Experiment Center, Institute of Basic Theory, Academy of Chinese Medical Sciences, 16 Nanxiaojie, Dongzhimennei, Beijing 100700, P.R. China

E-mail:maggie.wj@126.com

Key words: microglia, neuron, geniposide, ginsenoside Rg1, synergistic mechanism protected neuronal cell viability and inhibited LDH leakage, suppressed the expression of N-methyl-D-aspartate receptor subunit 1 and activated caspase-3, increased the mitochondrial transmembrane potential and improved the mitochondrial ultrastructure. MG-CM from separately used geniposide or ginsenoside $\operatorname{Rg} 1$ demonstrated differential neuroprotection at different levels. These findings revealed that the synergistic drug combination of geniposide and ginsenoside Rg1 in the treatment of stroke is a feasible approach for use.

\section{Introduction}

As the third leading cause of mortality worldwide, stroke is a major health issue in the elderly population as it leads not only to physical impairment, but also to a high risk of disability and social handicap (1). Evidence from preclinical studies (2) and randomized control trials (3) have demonstrated that combination therapy for ischemic stroke provides a survival advantage and increases the effectiveness of treatment without substantial side effects. Chinese herbs or their pharmacodynamic constituents have been widely used for the treatment of ischemic cerebral vascular disease through combination therapies (4). However, the complexity of the chemical ingredients has led to a substantial bottleneck in determining the mechanism of interaction among the ingredients for treating ischemic stroke (5).

Microglia, the resident immune cells of the central nervous system, have been implicated in triggering signaling cascades that lead to cell death in brain ischemic diseases (6). In physiological conditions, resting microglial processes make brief and direct contacts with neuronal synapses (7). In addition, microglial cells are restrained by numerous microenvironmental inhibitory effects, several of which are produced by neurons $(8,9)$. Neuron-microglial-cell inhibitory signaling is mediated by interactions, including CD200-CD200 receptor and CD22-CD45, also termed PTPRC (10). In pathological conditions, neuronal degeneration and microglial activation following transient cerebral ischemia (11) can effect the survival of neural cells through several pathways or cause neuronal injury $(12,13)$. Therefore, the inhibition of activated microglia may promote the survival of neurons. 
Geniposide and ginsenoside Rg1 (Fig. 1) are bioactive compounds, which are extracted from Cape Jasmine Fruit (Fructus Gardeniae) and Sanchi (Radix Notoginseng), respectively (14), and are two Chinese medicines, which have been used for the treatment of stroke for thousands of years $(15,16)$. Although the pharmacological mechanism of the individual use of geniposide or ginsenoside $\mathrm{Rg} 1$ on cerebral ischemia are well understood $(17,18)$, current understanding of the effect of the combined use of geniposide and ginsenoside Rg1 on stroke remains limited. Our previous study demonstrated that the combination of geniposide and ginsenoside $\mathrm{Rg} 1$, prescribed as a Tongluo Jiunao injection, can reduce the expression of macrophage inflammatory protein (MIP)-1 $\beta$ and C-C chemokine receptor type 5 (CCR5) in oxygen-glucose deprivation (OGD)-injured microglial cells (MCs), as well as inhibit the proliferative activity of microglial cells, suggesting the therapeutic potential of the combination of geniposide and ginsenoside Rg1 on ischemic cerebral ischemia (19). However, the synergistic mechanism of multi-component combined use for complex diseases remains to be fully elucidated. Synergistic therapeutic effects can be obtained from combining effective components from Chinese herbs through cell-cell communication. The present study, based on microglia-neuron communication, aimed to determine the synergistic effect of geniposide and ginsenoside Rg1 on hypoxia-injured neurons through treatment in differently treated microglial cell-conditioned media (MC-CM).

\section{Materials and methods}

BV2 microglia and N2a neuronal cells culture. The murine BV2 microglia cells and N2a neuronal cells (obtained from the Cerebrovascular Diseases Research Institute, Xuanwu Hospital of Capital Medical University, Beijing, China; third passage) were grown in T-25 tissue culture cell flasks at $5 \% \mathrm{CO}_{2}$ and $37^{\circ} \mathrm{C}$ humidified atmosphere using Dulbecco's modified Eagle's medium (DMEM)/F12 (Invitrogen Life Technologies, Carlsbad, CA, USA) culture media, supplemented with $10 \%$ fetal bovine serum (FBS; GE Healthcare Life Sciences, Logan, UT, USA), $2 \mathrm{mM}$ glutamine and $100 \mu \mathrm{g} / \mathrm{ml}$ penicillin-streptomycin. The BV2 microglial cells and N2a neuronal cells were maintained via two to three passages each week.

Establishment of the OGD model, drug administration and preparation of different microglial cell-conditioned media. An OGD model was used to mimic ischemia, as described in our previous study (20). For the microglial cells or N2a neuronal cells in OGD, culture medium was replaced with glucose-free DMEM, and the culture flasks (or plates) were placed into a sealed tank with a persistent low-flow (1.5 $1 / \mathrm{min}) 95 \% \mathrm{~N}_{2}$ and $5 \% \mathrm{CO}_{2}$ mixture to expire the oxygen for $20 \mathrm{~min}$. The inlet and outlet ends of the tubes were then clipped, and the tank was placed into an incubator for $6 \mathrm{~h}$ to mimic ischemia.

Geniposide (purity $>98 \%$; batch. no. 110749-200714) and ginsenoside $\operatorname{Rg} 1$ (purity>95\%, batch. no. 110703-201027) were chemically standardized products obtained from the National Institutes for Food and Drug Control (Beijing, China), which were validated using fingerprint chromatographic methodologies, according to the manufacturer's instructions. The microglial cells were divided into the following five groups: Control group; model group; geniposide-treated group (geniposide; $25 \mu \mathrm{g} / \mathrm{ml}$ ); ginsenoside Rg1-treated group (ginsenoside $\mathrm{Rg} 1 ; 5 \mu \mathrm{g} / \mathrm{ml}$ ); and combination-treated group (geniposide and ginsenoside $\operatorname{Rg} 1$ at a ratio of $1: 1)$. The microglial cells $\left(1 \times 10^{6}\right.$ cells $\left./ \mathrm{ml}\right)$ were preconditioned with the different drug treatments for $2 \mathrm{~h}$ and were maintained for another $6 \mathrm{~h}$ in hypoxia. The conditioned media from the five groups were collected and centrifuged at $1,000 \times \mathrm{g}$ for $10 \mathrm{~min}$ at $4^{\circ} \mathrm{C}$ to remove cell debris for the subsequent experiments.

The N2a neuronal cells were divided into seven groups: Control group (C), in which N2a neuronal cells were cultured in normal culture medium; model (M) group, in which N2a neuronal cells were challenged by OGD; N-MG-CM group, in which $\mathrm{N} 2$ a neuronal cells were cultured in normal cultured microglial cell-conditioned medium;I-MG-CM group: N2a neuronal cells were OGD-injured and cultured in microglial cell-conditioned medium; C-MG-CM group, in which $\mathrm{N} 2 \mathrm{a}$ neuronal cells were OGD-injured and cultured in geniposide and ginsenoside Rg1-treated microglial cell-conditioned medium; G-MG-CM group, in which N2a neuronal cells were OGD injured and cultured in geniposide-treated microglial cell-conditioned medium; R-MG-CM: N2a neuronal cells were OGD-injured and cultured in ginsenoside Rg1-treated microglial cell-conditioned medium. The proportion of conditioned media in each group was $100 \%$ and the incubation duration in the different conditioned media was $6 \mathrm{~h}$, according to our previous study (21).

CCK- 8 assay. $\mathrm{N} 2$ a neuronal cells at $1 \times 10^{3}$ cells per well were seeded onto 96 -well plates. Following incubation with the different microglial cell-conditioned media, the media in the 96-well culture plates were replaced with DMEM/F12 to avoid background interference. Subsequently, $10 \mu \mathrm{l} \mathrm{CCK-8)(Dojindo}$ Molecular Technologies, Inc., Kumamoto, Japan) was added to each well and incubated for $2 \mathrm{~h}$ at $37^{\circ} \mathrm{C}$, followed by measurement using a microplate reader (Safire ${ }^{2}$; TecanGroup, Ltd., Männedorf, Switzerland) with a test wavelength of $450 \mathrm{~nm}$ and a reference wavelength of $620 \mathrm{~nm}$.

Lactate dehydrogenase $(\mathrm{LDH})$ assay. $\mathrm{N} 2$ a neuronal cells at $1 \times 10^{3}$ cells/well were seeded onto 96-well plates. A CytoTox assay kit (Promega Corporation, Madison, WI, USA) was used for the enzymatic assessment of LDH release in the N2a neuronal cells. Reagents (substrate mixture and analysis buffer) were added into the 96-wells, according to the manufacturer's instructions. A fluorescence emission of $590 \mathrm{~nm}$ was used for measurement using a microplate reader. The rate of LDH leakage was calculated, according to the optical density (OD) values, using the following equation: $L L R=(O D$ value of the medium supernatant / OD value of the lysed cell supernatant) $\mathrm{x} 100 \%$.

Western blot analysis. N2a neuronal cells at $1 \times 10^{5}$ cells/well were seeded onto 6-well plates. Western blot analysis was performed to quantify the protein expression levels of N-methyl-D-aspartate receptor subunit 1 (NMDAR1) and activated caspase-3 (Abcam, Cambridge, UK) in the N2a neuronal cells. In brief, the N2a neuronal cells were washed with ice-cold PBS and scraped in lysis buffer (Beyotime Institute 
A

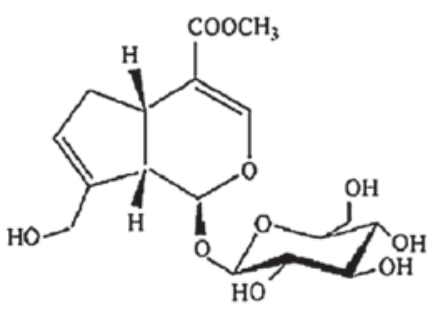

B<smiles>CC=C(C)C</smiles>

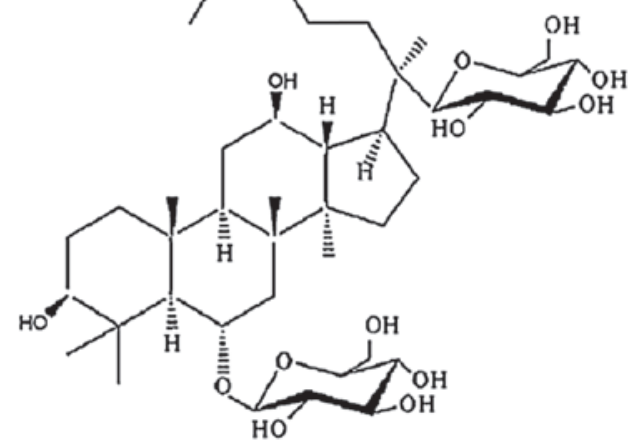

Figure 1. Chemical structure of (A) geniposide (C17H24O10; molecular weight, 300) and (B) ginsenoside Rg1 (C42H72O14; molecular weight, 800).

of Biotechnology, Jiangsu, China) comprised of $50 \mathrm{mM}$ Tris and $150 \mathrm{mM} \mathrm{NaCl}$ (TBS; pH 7.4), containing 1\% Triton X-100, $1 \%$ Nonidet P-40, 0.5\% sodium-deoxycholate, $0.1 \%$ sodium-dodecyl-sulfate, $1 \mathrm{mM}$ phenylmethylsulfonyl fluoride, $15 \mu \mathrm{g} / \mathrm{ml}$ leupeptin, $71 \mu \mathrm{g} / \mathrm{ml}$ phenanthrolyne and $20 \mathrm{U} / \mathrm{ml}$ aprotine. The insoluble material was removed by centrifugation at $9,500 \mathrm{xg}$ for $20 \mathrm{~min}$ at $4^{\circ} \mathrm{C}$. The protein content was measured, according to the bicinchoninic acid method (WellBiz Brands, Inc., Highlands Ranch, CO, USA). Subsequently, $20 \mu \mathrm{g}$ protein was processed using SDS-PAGE separation on $12.5 \%$ polyacrylamide gel, and transferred onto a $0.45 \mu \mathrm{m}$ nitrocellulose membrane (Pierce Biotechnology, Inc., Rockford, IL, USA). Non-specific binding sites were blocked with TBS, comprised of $40 \mathrm{mM}$ Tris, (pH 7.6) and $300 \mathrm{mM} \mathrm{NaCl}$, containing 5\% nonfat dry milk, for $1 \mathrm{~h}$ at $37^{\circ} \mathrm{C}$. The membrane was then incubated with the following antibodies: Rabbit polyclonal anti-NMDAR1 (1:500; cat. no. ab17345; Abcam) and rabbit polyclonal anti-activated caspase-3 (1:500; cat. no. ab2302; Abcam) overnight at $4^{\circ} \mathrm{C}$, followed by incubation in a 1:5,000 dilution of horseradish-peroxidase-conjugated goat anti-rabbit IgG (cat. no. ZB-2301; ZSGB-BIO, Beijing, China) at $37^{\circ} \mathrm{C}$ for $1 \mathrm{~h}$. Immunoreactive proteins were detected by enhanced chemiluminescence (Pierce Biotechnology, Inc.), according to the manufacturer's instructions. The membrane was then incubated with stripping buffer (Applygen Technologies Inc, Beijing, China) for $0.5 \mathrm{~h}$ at room temperature, followed by incubation with rabbit polyclonal anti- $\beta$-actin $(1: 5,000$; cat. no. ab119716; Abcam) and the corresponding secondary antibody. The experiment was repeated in triplicate, and three wells were used for each group.

Analysis of mitochondrial transmembrane potential. N2a neuronal cells at $1 \times 10^{3}$ cells/well were seeded onto 24 -well plates. The mitochondrial membrane potential was investigated using a JC-1 probe (Beyotime Institute of Biotechnology), which exists either as a green fluorescent monomer at depolarized membrane potentials or as a red fluorescent J-aggregate at hyperpolarized membrane potentials. The JC-1 was added into the 24-wells, according to the manufacturer's instructions. Fluorescent images were captured and the ratios of the mitochondrial aggregates (red) to the monomeric form of JC-1 (green) were analyzed using fluorescence microscopy (Nikon Eclipse 80i; Nikon Corporation, Tokyo, Japan).
Transmission electron microscopy. N2a neuronal cells at $1 \times 10^{3}$ cells/well were seeded onto 24 -well plates. The mitochondrial changes of the N2a neuronal cells were observed using transmission electron microscopy. In brief, the N2a neuronal cells were fixed with $4 \%$ glutaraldehyde and $1 \%$ osmic anhydride in sequence, and then dehydrated with acetone (Sigma-Aldrich, St. Louis, MO, USA). Subsequently, 50-70 nm ultrathin sections were cut using an ultramicrotome (LKB, Margate, FL, USA) and stained with $2 \%$ uranyl acetate (Sigma-Aldrich). Transmission electron microscopy (H7650; Hitachi, Ltd., Tokyo, Japan) was used to observe the autophagosome in the cells.

Statistical analysis. All results are expressed as the mean \pm standard deviation. SPSS 13.0 software (SPSS, Inc., Chicago, IL, USA) was used for statistical analysis. One way analysis of variance was used to determine statistically significant differences among groups. $\mathrm{P}<0.05$ was considered to indicate a statistically significant difference.

\section{Results}

$N 2$ a neuronal cellular viability and LDH leakage. The cellular viability and LDH leakage of the N2a neuronal cells incubated with the different MG-CM were assessed using a CCK-8 assay and LDH kits. Compared with the control group, the viability of the N2a neuronal cells in the model group was reduced significantly by OGD injury $(\mathrm{P}<0.001$; Fig. 2A), while LDH leakage in the N2a neuronal cells in the model group was increased significantly following challenge by OGD injury $(\mathrm{P}<0.001$; Fig. $2 \mathrm{~B})$, which indicated that the N2a neuronal cells were injured by OGD. No significant change in viability or LDH leakage were observed in the N-MG-CM group, compared with control group, which indicated that the microglial cells had no effect on the normal cultured N2a neuronal cells. The viability was reduced and LDH leakage was increased in the N2a neuronal cells incubated with I-MG-CM, with more severe injury, compared with the group exposed to OGD injury alone. The viability and LDH leakage of the N2a neuronal cells incubated with C-MG-CM, G-MG-CM and R-MG-CM were recovered to different extents. There were different effects of the MG-CM of geniposide and ginsenoside $\mathrm{Rg} 1$ on the $\mathrm{N} 2 \mathrm{a}$ neuronal 
A

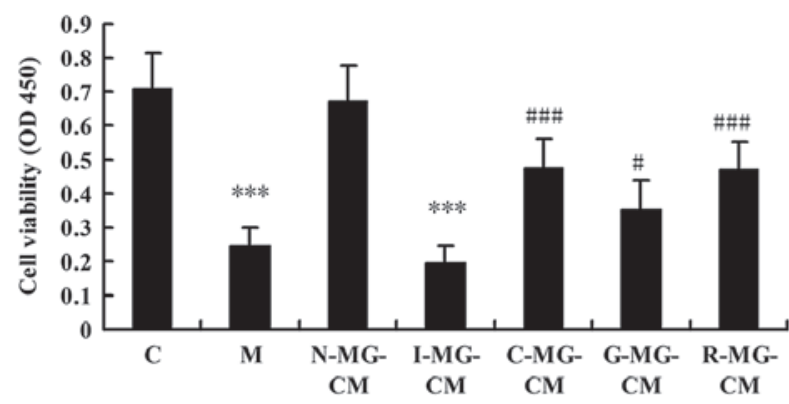

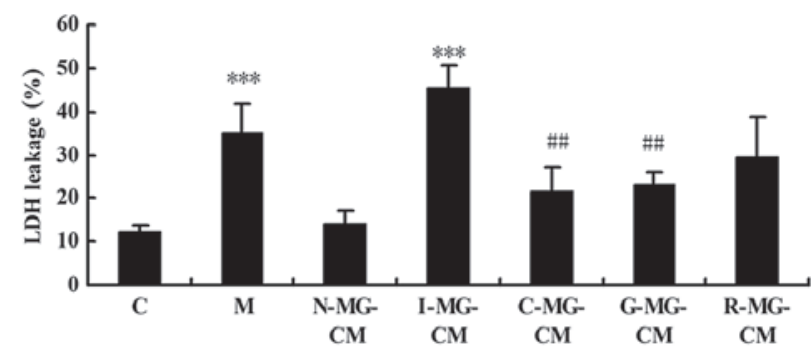

Figure 2. (A) Bar graphs indicating the changes in neuronal cell viability, determined using a Cell Counting Kit-8 assay. A significant decrease in cell viability was observed in the neuronal cells in the M or I-MG-CM groups, and this cell viability was recovered when the neuronal cells were cultured with G-MG-CM, R-MG-CM or C-MG-CM, with the R-MG-CM and C-MG-CM having the same effect. (B) Bar graphs indicating the changes of LDH leakage in the neuronal cell cultural media. The LDH leakage in the M or I-MG-CM group was significantly increased, compared with the control (C) group. The LDH leakage in the C-MG-CM and G-MG-CM groups were suppressed, compared with the $\mathrm{M}$ group. No effect was observed in the R-MG-CM group. ${ }^{* * *} \mathrm{P}<0.001$, vs. $\mathrm{C}$ group; ${ }^{\#} \mathrm{P}<0.05$, vs. M group; ${ }^{\# \#} \mathrm{P}<0.01$, vs. $\mathrm{M}$ group; ${ }^{\# \# \#} \mathrm{P}<0.001$, vs. $\mathrm{M}$ group. Data are expressed as the mean \pm standard deviation. $\mathrm{C}$, control; $\mathrm{M}$, model (ischemia); MG-CM, microglial cell conditioned medium; C-MG-CM, combined geniposide and ginsenoside Rg1 MG-CM; G-MG-CM, geniposide MG-CM; R-MG-CM, ginsenoside Rg1 MG-CM; OD, optical density; LDH, lactate deydrogenase.
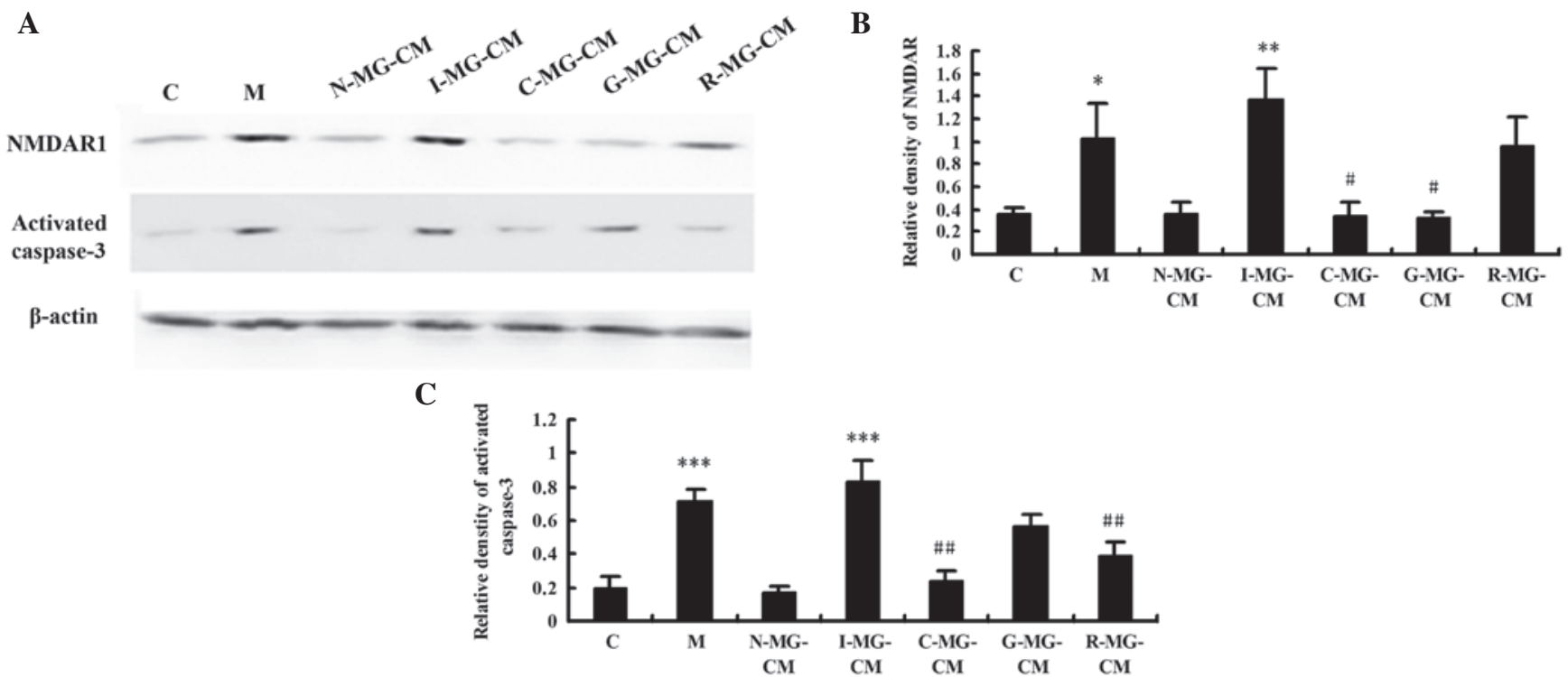

Figure 3. (A) Expression levels of NMDAR1 and activated caspase-3 in neuronal cells, assessed using western blotting. Images show representative blots of NMDAR1, activated caspase-3 and $\beta$-actin (loading control). (B and C) Bar graphs indicate the relative densities of the NMDAR1 and activated caspase-3 bands, estimated quantitatively using Phoretix 1D image software. Values represent the mean optical density ratio relative to the loading control. ${ }^{*} \mathrm{P}<0.05$, vs. C group; ${ }^{* * * *} \mathrm{P}<0.001$, vs. $\mathrm{C}$ group; ${ }^{*} \mathrm{P}<0.05$, vs. M group; ${ }^{\# *} \mathrm{P}<0.01$, vs. $\mathrm{M}$ group. Data are expressed as the mean \pm standard deviation. $\mathrm{C}$, control; $\mathrm{M}$, model (ischemia); MG-CM, microglial cell conditioned medium; C-MG-CM, combined geniposide and ginsenoside Rg1 MG-CM; G-MG-CM, geniposide MG-CM; R-MG-CM, ginsenoside Rg1 MG-CM.

cells. In terms of cell viability, the effect of the ginsenoside Rg1-treated MG-CM was more marked than that of geniposide. For the LDH leakage improvement, the effect of the geniposide-treated MG-CM was more marked than that of ginsenoside Rg1. Incubation with MG-CM with the combined use of geniposide and ginsenoside $\operatorname{Rg} 1$ improve cell viability and suppressed LDH leakage.

Expression of NMDAR1 and activated caspase-3 in N2a neuronal cells. The present study subsequently investigated the expression levels of NMDAR1 and activated caspase-3 in he N2a neuronal cells in the different groups. As shown in Fig. 3, NMDAR1 and activated caspase-3 in the N2a neuronal cells exhibited a significant increase in expression in the model group (2.89-fold, $\mathrm{P}<0.05$ and 3.73-fold, $\mathrm{P}<0.001$, respectively) and I-MG-CM group (3.85-fold, $\mathrm{P}<0.01$; 4.34-fold, $\mathrm{P}<0.001$, respectively), compared with the control group, indicating that injury and apoptosis were induced by OGD and I-MG-CM. Compared with the model group, the use of MG-CM with ginsenoside Rg1 alone had no effect on the expression of NMDAR1. Marked suppression of the expression of NMDAR1 was observed in the N2a neuronal cells incubated in MG-CM with ginsenoside Rg1 alone, the effect of which was more marked than that of MG-CM with geniposide and ginsenoside combined $(\mathrm{P}<0.05)$. In terms of the expression of activated caspase-3, the effect of MG-CM with combined use of geniposide and ginsenoside was the same as that observed in the R-MG-CM group, with a clear reduction. The protein level of activated caspase- 3 in the R-MG-CM group was unaffected relative to the model group. 

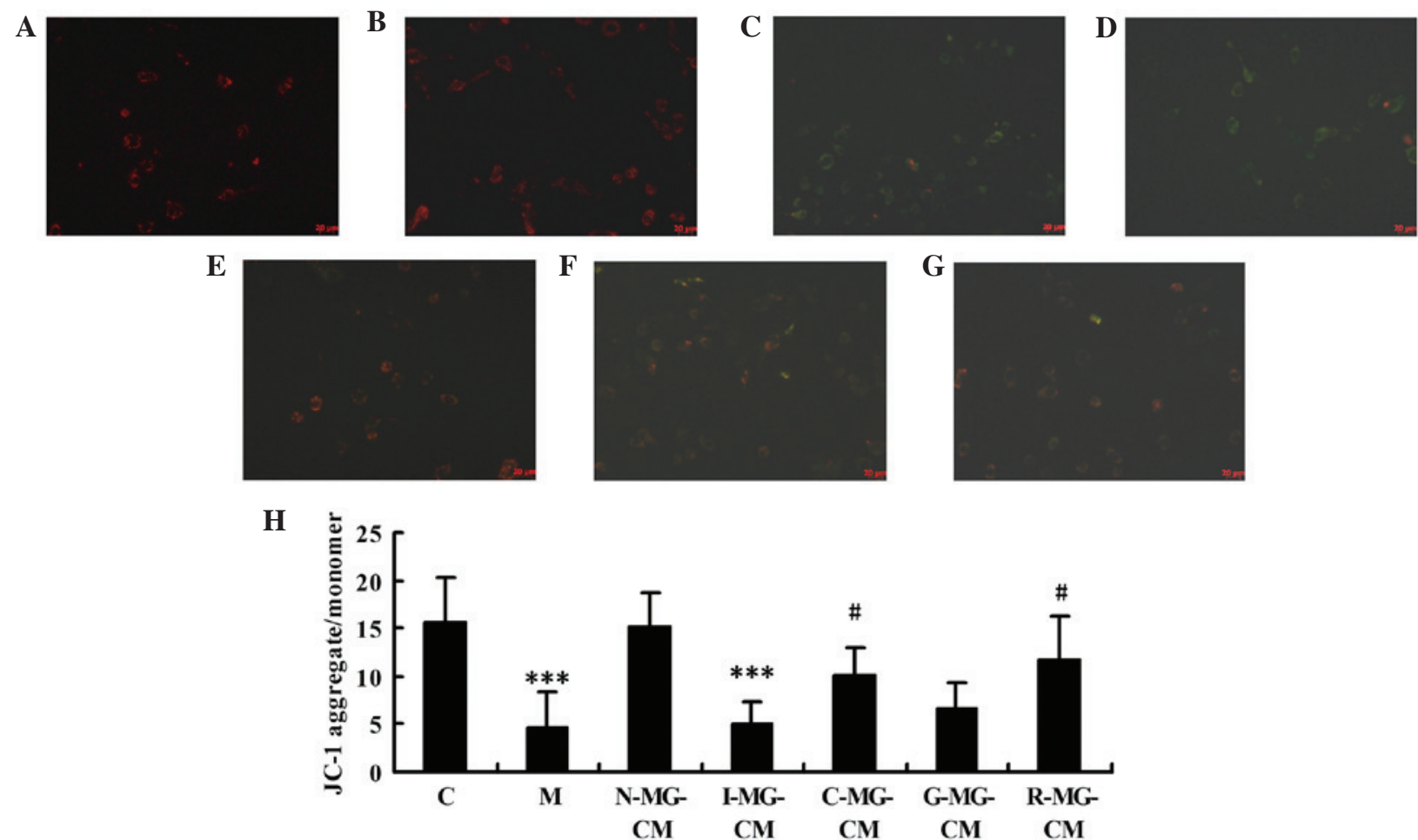

Figure 4. OGD- or OGD-injured MG-CM-induced mitochondrial membrane potential loss is prevented by ginseonside Rg1 or the combined use of geniposide and ginsenoside $\mathrm{Rg} 1$. A using a JC-1 probe was used to examine the mitochondrial membrane potential in neuronal cells, which were (A) untreated or were treated with (B) N-MG-CM, (C) OGD, (D) I-MG-CM, (E) C-MG-CM, (F) G-MG-CM or (G) R-MG-CM for 6 h. Magnification, x400. (H) Quantitative presentation of the JC-1 assay. ${ }^{* * *} \mathrm{P}<0.001$, vs. C group, ${ }^{\#} \mathrm{P}<0.05$ vs. M group. Results are expressed as the mean \pm standard deviation of triplicate determinations. C, control; M, model (ischemia); OGD, oxygen-glucose deprivation; MG-CM, microglial cell conditioned medium; N-MG-CM, normal MG-CM; C-MG-CM, combined geniposide and ginsenoside Rg1 MG-CM; G-MG-CM, geniposide MG-CM; R-MG-CM, ginsenoside Rg1 MG-CM.

Mitochondrial transmembrane potential of N2a neuronal cells. Almost all the cells visualized were well-spread and exhibited red or orange fluorescence in the untreated N2a neuronal cells (Fig. 4A) and cells in the N-MG-CM group (Fig. 4B). By contrast, in the OGD or I-MG-CM groups (Fig. 4C and D), the majority of fluoresced green exclusively, indicating loss of mitochondrial membrane potential. The N2a neuronal cells incubated with C-MG-CM, G-MG-CM or R-MG-CM exhibited red or orange fluorescence, which indicated an improvement relative to the model group, with C-MG-CM being the most similar to the control cells (Fig. 4E-G). Ratios of JC-1 aggregates/monomeric forms in the groups (Fig. $4 \mathrm{H}$ ) indicated that the OGD- or I-MG-CM-induced loss of N2a neuronal cell mitochondrial membrane potential was prevented by MG-CM with geniposide and/or ginsenoside.

Mitochondrial changes in the N2a neuronal cells. The mitochondrial structure of the $\mathrm{N} 2 \mathrm{a}$ neuronal cells in the control group was evident, with an intact mitochondrial membrane and mitochondrial crista (Fig. 5A). There was no change to the mitochondrial structure of the cells treated with N-MG-CM (Fig. 5B). By contrast, OGD (Fig. 5C) or I-MG-CM (Fig. 5D) incubation damaged the N2a neuronal cells mitochondrial structure, characterized by disordered mitochondrial crista arrangement or vacuolation. Following incubation with $\mathrm{C}-\mathrm{MG}-\mathrm{CM}, \mathrm{G}-\mathrm{MG}-\mathrm{CM}$ or R-MG-CM, the mitochondrial structure of the N2a neuronal cells recovered to different extents, compared with the model group, with the G-MG-CM group and C-MG-CM group exhibiting more significant effects (Fig. 5E and F).

\section{Discussion}

Pharmacodynamic constituents from natural medicines have been investigated for the treatment of ischemic stroke. Multi-component treatments, characterized by two or more agents interacting with multiple targets simultaneously, are considered to be a rational and efficient form of therapy that is designed to control complex diseases $(22,23)$, including stroke, which is a more complex disease than initially anticipated. Our previous study and those of others have already demonstrated that multi-component prescription (Tongluo Jiunao injection), composed of ginsenoside and ginsenoside $\mathrm{Rg} 1$ is effective for the treatment of stroke due to its anti-inflammatory, neuronprotecive and neurotrophic roles $(19,24)$. The present study aimed to elucidate the synergistic effects of microglial cell-conditioned media treated with geniposide and ginsenoside $\operatorname{Rg} 1$ on hypoxia-injured neurons.

According to the results obtained from the CCK-8 assay and LDH leakage assessment, certain proteins secreted from the OGD-activated microglial cells were involved in the neuronal cell damage, which indicated that the OGD-induced microglial cells generated neurotoxicity. An improvement in the I-MG-CM-induced increase of LDH was observed by using G-MG-CM, while cell viability enhancement was not significantly difference. R-MG-CM increased the neuronal cell viability, however had no effect on LDH leakage, suggesting that microglial cells treated by ginsenoside Rg1 alone improved neuron survival by secreting certain cytokines through pathways other than LDH. MG-CM with the 


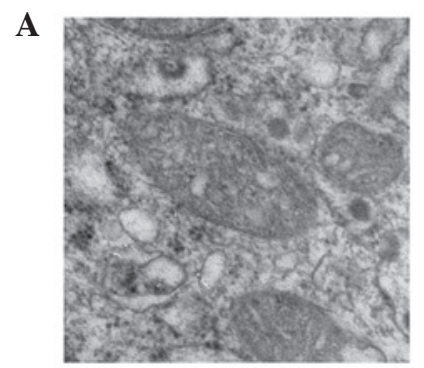

B

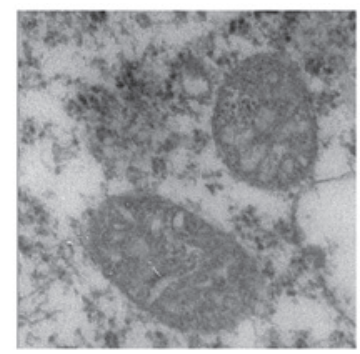

C
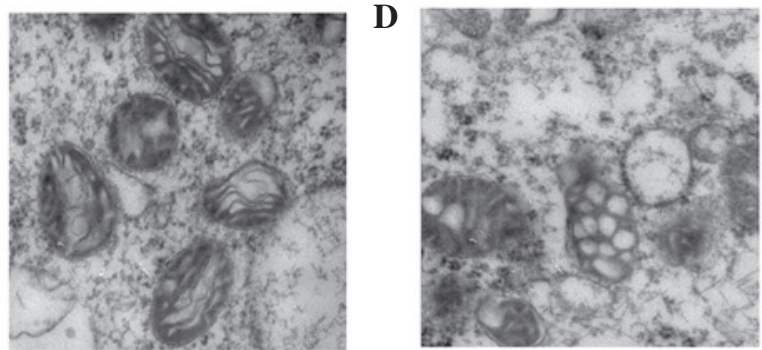

$\mathbf{E}$

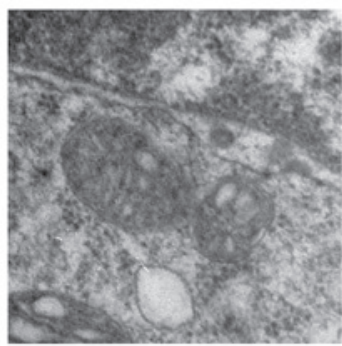

$\mathbf{F}$
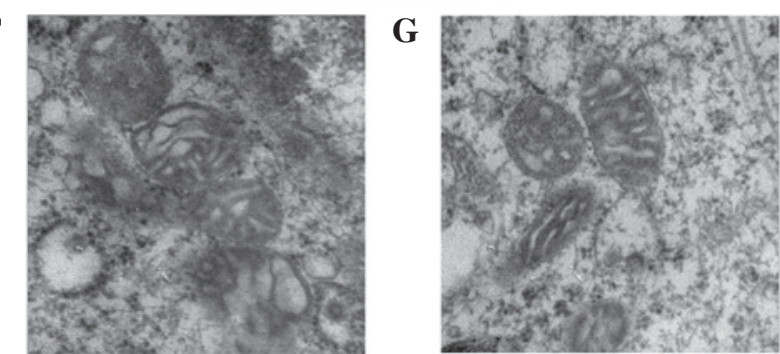

Figure 5. OGD- or OGD-injured MF-CM-induced mitochondrial abnormalities are prevented by treatment with MG-CM with individual or combined use of geniposide and ginsenoside Rg1. Ultrastructural features of mitochondrial change in neuronal cells in the (A) untreated control, or following treatment with (B) N-MG-CM, (C) OGD, (D) I-MG-CM, (E) C-MG-CM, (F) G-MG-CM or (G) R-MG-CM for 6 h. Magnification, x80,000. OGD, oxygen-glucose deprivation; C, control; M, model (ischemia); MG-CM, microglial cell conditioned medium; C-MG-CM, combined geniposide and ginsenoside Rg1 MG-CM; G-MG-CM, geniposide MG-CM; R-MG-CM, ginsenoside Rg1 MG-CM.

combined use of ginsenoside and ginsenoside $\mathrm{Rg} 1$ increased the cell viability and decreased LDH leakage, which demonstrated an integral effect of compatibility.

Activated-microglia release glutamate, which is the major neurotoxic factor released into the extracellular space following neural injury and causes neuronal death at high concentrations (25). Glutamate activates ionotropic and metabotropic receptors, and NMDA is a type of ionotropic receptor. The action of glutamate causes $\mathrm{Na}^{+}$and $\mathrm{Ca}^{2+}$ influx, which can lead to $\mathrm{Ca}^{2+}$ overload and subsequent $\mathrm{Ca}^{2+}$ dependent neural injury $(26,27)$. Another important mechanism of OGD-injury in neural cells is apoptosis. Glutamate can also induce the loss of neurons and activation of caspase-3 (28), a key apoptotic protease-mediated cascade downstream. Several reports have demonstrated that cytochrome $c$-dependent caspase- 3 activation is an important mechanism responsible for ischemia-induced apoptosis $(29,30)$.

Consequently, the present study hypothesized that the secretion of microglia can be altered due to geniposide and ginsenoside $\operatorname{Rg} 1$ compatibility, to improve the survival of the neuronal microenvironment. The results demonstratedthat microglial cells, following treatment with geniposide, downregulated the expression of the NMDA receptor in neurons, which indicated that geniposide inhibited the expression of NMDA receptors by reducing the glutamate secretion of the ischemic microglia. The above effect was almost absent following treatment with ginsenoside $\mathrm{Rg} 1$, however downregulated of caspased-3 was observed, which indicated an anti-apoptotic effect. By contrast, the MG-CM with geniposide alone had no effect on caspase-3, suggesting that other possible neuroprotective pathways were involved, including anti-inflammatory or caspase-independent pathways. Treatment with MG-CM with geniposide and ginsenoside Rg1 in combination suppressed the expression of NMDA receptor and caspase-3, which demonstrated integrated and synergistic modulation due to the compatibility of the effective components.
Mitochondria are one of the important pathways of neuronal cells apoptosis. Several studies have demonstrated that OGD induces apoptosis by favoring the release of cytochrome $c$ and the consequent formation of the apoptotic complex $(31,32)$. In the present study, when the neuronal cells were exposed to OGD or I-MG-CM, rapid depolarization of the mitochondrial membrane potential was observed, which is indicative of mitochondrial dysfunction. The above mitochondrial dysfunction was observed in the electron microscopy images, characterized by mitochondrial swelling and osmotic expansion, suggestive of typical mitochondrial pathological change (33). The observed mitochondrial dysfunction was alleviated by treatment with MG-CM with geniposide and ginsenoside Rg1 in combination, which indicated that the synergetic use of geniposide and ginsenoside Rg1 suppressed OGD-induced neuronal apoptosis through inhibition of the mitochondrial-mediated apoptotic pathway. Of note, it was observed that, compared with treatment with geniposide alone, ginsenoside Rg1 exhibited a more significant effect on the improvement of mitochondrial membrane potential and mitochondrial ultrastructure. Combined with the results obtained on the expression of caspase-3, it was hypothesized that, in the synergistic effect of geniposide and ginsenoside Rg1, ginsenoside $\operatorname{Rg} 1$ is the predominant effector in the inhibition of the mitochondrial-mediated apoptotic pathway.

From the above-mentioned results, it appears that paracrine signaling in differently treated microglia are involved in neuroprotection. However, which signal is elicited by geniposide and/or ginsenoside Rg1, and how the synergistic effect produces the observed results remains to be fully elucidated. Our previous study indicated that the synergistic use of geniposide and ginsenoside Rg1 can balance microglial TNF- $\alpha$ and TGF- $\beta 1$ following ischemic injury (34). The balance of microglial neurotoxic factors and the neuroprotective factor, Rg1, may explain the neuroprotective effects observed following geniposide and ginsenoside synergistic use. 
In conclusion, the present study demonstrated that MG-CM with geniposide and ginsenoside Rg1 in combintation exerted a synergistic effect on the neuronal mitochondrial-mediated apoptotic pathway triggered by OGD, with geniposide and ginsenoside Rg1 exhibiting different regulatory effects.

\section{Acknowledgements}

This study was supported by grants from the National Natural Science Foundation of China (grant. nos. 81102679 and 81473449), the Fundamental Research Funds for the Central Public Welfare Research Institutes (grant no. ZZ070824), and the National Basic Research Program of China (973 Program, 2015CB554400).

\section{References}

1. Feigin VL, Lawes CM, Bennett DA, Barker-Collo SL and Parag V: Worldwide stroke incidence and early case fatality reported in 56 population-based studies: A systematic review. Lancet Neurol 8: 355-369, 2009.

2. Faure S, Oudart N, Javellaud J, Fournier A, Warnock DG and Achard JM: Synergistic protective effects of erythropoietin and olmesartan on ischemic stroke survival and post-stroke memory dysfunctions in the gerbil. J Hypertens 24: 2255-2261, 2006.

3. Smadja D, Chausson N, Joux J, Saint-Vil M, Signaté A, Edimonana M, Jeannin S, Bartoli B, Aveillan M, Cabre P and Olindo S: A new therapeutic strategy for acute ischemic stroke: Sequential combined intravenous tpa-tenecteplase for proximal middle cerebral artery occlusion based on first results in 13 consecutive patients. Stroke 42: 1644-1647, 2011

4. Zhang J, Li P, Wang Y, Liu J, Zhang Z, Cheng W and Wang Y: Ameliorative effects of a combination of baicalin, jasminoidin and cholic acid on ibotenic acid-induced dementia model in rats. PLoS One 8: e56658, 2013.

5. Liu J, Zhou CX, Zhang ZJ, Wang LY, Jing ZW and Wang Z: Synergistic mechanism of gene expression and pathways between jasminoidin and ursodeoxycholic acid in treating focal cerebral ischemia-reperfusion injury. CNS Neurosci Ther 18: 674-682, 2012

6. Lai AY and Todd KG: Microglia in cerebral ischemia: Molecular actions and interactions. Can J Physiol Pharmacol 84: 49-59, 2006.

7. Wake H, Moorhouse AJ, Jinno S, Kohsaka S and Nabekura J: Resting microglia directly monitor the functional state of synapses in vivo and determine the fate of ischemic terminals. J Neurosci 29: 3974-3980, 2009.

8. Ransohoff RM and Perry VH: Microglial physiology: Unique stimuli, specialized responses. Annu Rev Immunol 27: 119-145, 2009.

9. Hanisch UK and Kettenmann H: Microglia: Active sensor and versatile effector cells in the normal and pathologic brain. Nature Neurosci 10: 1387-1394, 2007.

10. Barclay AN, Wright GJ, Brooke G and Brown MH: CD200 and membrane protein interactions in the control of myeloid cells. Trends Immunol 23: 285-290, 2002.

11. Moon JB, Lee CH, Park CW, Cho JH, Hwang IK, Yoo KY, Choi JH, Shin HC and Won MH: Neuronal degeneration and microglial activation in the ischemic dentate gyrus of the gerbil. J Vet Med Sci 71: 1381-1386, 2009.

12. Taylor DL, Pirianov G, Holland S, McGinnity CJ, Norman AL, Reali C, Diemel LT, Gveric D, Yeung D and Mehmet H: Attenuation of proliferation in oligodendrocyte precursor cells by activated microglia. J Neurosci Res 88: 1632-1644, 2010.

13. Hur J, Lee P, Kim MJ, Kim Y and Cho YW: Ischemia-activated microglia induces neuronal injury via activation of gp91phox NADPH oxidase. Biochem Biophys Res Commun 391: 1526-1530, 2010

14. Liu Y, Hua Q, Lei H and Li P: Effect of Tong Luo Jiu Nao on $\mathrm{A} \beta$-degrading enzymes in AD rat brains. J Ethnopharmacol 137: 1035-1046, 2011.

15. Li C, Pan Y, Jia X, Li K, Liu C, Wang X and Li P: Effects of Huanqin (dried root of Scutellaria baicalensis) and Zhizi (dried fruit of Gardenia jasminoides) used in combination on ischemic cascade reaction in the rat model of focal cerebral ischemia and reperfusion. Beijing Zhong Yi Yao Da Xue Xue Bao 25: 31-33, 2002 (In Chinese).
16. Li K: Systematic review of Sanqi agents for acute ischaemic stroke. Lin Chuang Hui Cui 22: 1-5, 2007 (In Chinese).

17. Chen J, Sun M, Wang X, Lu J, Wei Y, Tan Y, Liu Y, Götz J, $\mathrm{He} \mathrm{R}$ and Hua Q: The herbal compound geniposide rescues formaldehyde-induced apoptosis in N2a neuroblastoma cells. Sci China Life Sci 57: 412-421, 2014.

18. Xie CL, Wang WW, Xue XD, Zhang SF, Gan J and Liu ZG: A systematic review and meta-analysis of Ginsenoside-Rg1 (G-Rg1) in experimental ischemic stroke. Sci Rep 5: 7790, 2015.

19. Wang J, Li PT, Du H, Hou JC, Li WH, Pan YS and Chen HC: Tong Luo Jiu Nao injection, a traditional Chinese medicinal preparation, inhibits MIP-1 $\beta$ expression in brain microvascular endothelial cells injured by oxygen-glucose deprivation. J Ethnopharmacol 141: 151-157, 2012.

20. Wang J, Hou J, Zhang P, Li D, Zhang C and Liu J: Geniposide reduces inflammatory responses of oxygen-glucose deprived rat microglial cells via inhibition of the TLR4 signaling pathway. Neurochem Res 37: 2235-2248, 2012.

21. Wang J, Li PT, Du H, Hou JC, Li WH, Pan YS, Hua Q and Chen HC: Impact of paracrine signals from brain microvascular endothelial cells on microglial proliferation and migration. Brain Res Bull 86: 53-59, 2011.

22. Carey KM, Comee MR, Donovan JL and Kanaan AO: A polypill for all? Critical review of the polypill literature for primary prevention of cardiovascular disease and stroke. Ann Pharmacother 46: 688-695, 2012.

23. Zimmermann GR, Lehár J and Keith CT: Multi-target therapeutics: When the whole is greater than the sum of the parts. Drug Discov Today 12: 34-42, 2007.

24. Li H, Xu J, Wang X and Yuan G: Protective effect of ginsenoside Rg1 on lidocaine-induced apoptosis. Mol Med Rep 9: 395-400, 2014.

25. Umebayashi D, Natsume A, Takeuchi H, Hara M, Nishimura Y, Fukuyama R, Sumiyoshi N and Wakabayashi T: Blockade of gap junction hemichannel protects secondary spinal cord injury from activated microglia-mediated glutamate exitoneurotoxicity. J Neurotrauma 31: 1967-1974, 2014.

26. Kawasaki Y, Kohno T, Zhuang ZY, Brenner GJ, Wang H, Van Der Meer C, Befort K, Woolf CJ and Ji RR: Ionotropic and metabotropic receptors, protein kinase $\mathrm{A}$, protein kinase $\mathrm{C}$, and Src contribute to C-fiber-induced ERK activation and cAMP response element-binding protein phosphorylation in dorsal horn neurons, leading to central sensitization. J Neurosci 24: 8310-8321, 2004

27. Novitskaya YA, Dravolina OA, Zvartau EE, Danysz W and Bespalov AY: Interaction of blockers of ionotropic NMDA receptors and metabotropic glutamate receptors in a working memory test in rats. Neurosci Behav Phsyiol 40: 807-811, 2010.

28. Xu GY, Liu S, Hughes MG and McAdoo DJ: Glutamate-induced losses of oligodendrocytes and neurons and activation of caspase- 3 in the rat spinal cord. Neuroscience 153: 1034-1047, 2008.

29. Yin J, Li H,Feng C and Zuo Z: Inhibition of brain ischemia-caused notch activation in microglia may contribute to isoflurane postconditioning-induced neuroprotection in male rats. CNS Neurol Disord Drug Targets 13: 718-732, 2014.

30. Yang $X$ and Huang N: Berberine induces selective apoptosis through the AMPK-mediated mitochondrial/caspase pathway in hepatocellular carcinoma. Mol Med Rep 8: 505-510, 2013.

31. Wang L, Chen M, Yuan L, Xiang Y, Zheng R and Zhu S: 14,15-EET promotes mitochondrial biogenesis and protects cortical neurons against oxygen/glucose deprivation-induced apoptosis. Biochem Biophys Res Commun 450: 604-609, 2014.

32. Wang J, Wang P, Li S, Wang S, Li Y, Liang N and Wang M: Mdivi-1 prevents apoptosis induced by ischemia-reperfusion injury in primary hippocampal cells via inhibition of reactive oxygen species-activated mitochondrial pathway. J Stroke Cerebrovasc Dis 23: 1491-1499, 2014.

33. Lee WK and Thévenod F: A role for mitochondrial aquaporins in cellular life-and-death decisions?. Am J Physiol Cell Physiol 291: C195-C202, 2006.

34. Wang J, Hou JC, Xiang LH, Zhang J and Ju DH: Compatibility of geniposide and ginsenoside $\mathrm{rg} 1$ : Their regulating roles in secretion of anoxia induction injured microglia inflammatory cytokines. Zhongguo Zhong Xi Yi Jie He Za Zhi 34: 91-95, 2014 (In Chinese). 